\title{
Modeling Air Conditioning System with Storage Evaporator for Vehicle Energy Management
}

\author{
Quansheng Zhang*, Marcello Canova \\ Center for Automotive Research, \\ The Ohio State University \\ 930 Kinnear Road, \\ Columbus, OH, 43212, United States
}

\begin{abstract}
Automotive Air Conditioning (A/C) system significantly affects fuel consumption and emission. Thus, Phase Change Material (PCM) is exploited in an innovative storage evaporator to improve the A/C system performance. Due to hybrid features introduced by mode switching when PCM changes its status between liquid and solid, the task of control-oriented modeling is particularly challenging. Upon the energy-based model built, optimal control of an advanced A/C system with a storage evaporator is formulated as to find an optimal clutch command sequence balancing fuel consumption, cabin comfort and drivability constraints. In the scope of vehicle energy management, Dynamic Programming (DP) algorithm usually serves as a tool of obtaining benchmark optimal solution against which results from other optimal algorithms are compared. However, a direct application of DP algorithm to the optimization problem faces unexpected difficulty, because the discretization of state space is not feasible for an irregular multi-dimensional subspace formed by the multi-mode model. Alternatively, hybrid optimal control theory is pursued and a preliminary study is conducted to illustrate its promising application.
\end{abstract}

Keywords: Air Conditioning System, Storage Evaporator, Phase Change Material, Dynamic Programming, Hybrid Optimal Control

\footnotetext{
* Corresponding Author

Email address: zhang.777@osu.edu (Quansheng Zhang)
}

Preprint submitted to Applied Thermal Engineering

April 1, 2015 


\section{Introduction}

Automotive A/C system has profound effects on the vehicle fuel economy. The majority of A/C system is still heuristically controlled and operates in an inefficient way. An analysis conducted in NREL [1] showed that the use of systems is equivalent to $5.5 \%$ of the domestic light duty vehicle fuel consumption. Two directions are mainly pursued in order to reduce fuel consumption of A/C system, namely model-based optimization and control design as well as hardware upgrades. The mass migration and heat transfer inside refrigerant loop is usually modeled using energy-based method [2], and its energy management is a typical a multi-objective optimization problem balancing fuel consumption, cooling requirement and mechanical weariness [3]. In [4-8], the optimal compressor clutch sequence is found using online implementable model predictive control (MPC) method. Recently, storage evaporator [9] is introduced to improve A/C system performance, because it increases thermal inertia and expands energy storage capability when the PCM changes its status between liquid and solid.

PCM has been widely applied in industrial areas, such as solar power plants,, electronic devices, and transport $[10,11]$. Depending on the specific application, different models have been developed to characterize the heat transfer and phase change dynamics. Generally, these models belong to two model categories, namely distributed-parameter model and lumped-parameter model. In [12-14], the phase change material is treated as a one dimensional heat transfer model, a partial differential equation with boundary conditions specified. In [15], a simplified dynamic model is developed for predicting the energy impact due to the addition of the PCM. Usually, these models are tailored for performance analysis at design stage, helping the designers determine appropriate geometric parameters or material types. However, little attention has been paid to the optimal operation of the PCM after the design stage.

This paper is aimed to apply optimal control theory on automotive A/C system with storage evaporator. This work is challenging as it requires the 
modeling of phase change dynamics and choice of appropriate control methods. The A/C loop dynamics changes significantly over PCM status, such as completely frozen, freezing/melting and completely melt. In other words, hybrid system dynamics exists and should be captured by the control-oriented model developed. The hybrid feature also affects the optimization process, as optimization algorithms applicable only in continuous domain might fail. Therefore, hybrid optimal control theory specifically developed for hybrid system is pursued herein. Different versions of Hybrid Minimum Principle (HMP) exist. A general version of the HMP is presented by Sussmann [16] based on a set of needle variations and a Boltyanski approximation cone. Two version of HMPs are introduced by Riedinger et al. [17, 18] and Shaikh and Caines [19] for hybrid systems with autonomous and controlled switching. Although the above HMPs have solid theoretical foundation, it has hardly been applied to a practical problem before, like the energy management of $\mathrm{A} / \mathrm{C}$ system. Therefore, this paper is aimed to illustrate the benefit of storage evaporator and the potential application of hybrid optimal theory on the A/C system energy management.

The paper is organized as follows. Section II models the A/C system dynamics using a lumped-parameter approach and resulted system equations are in descriptor form. Section III compares the performances between storage evaporator and conventional evaporator, and identifies the benefits of enhanced thermal inertia. Section IV fits the problem of optimal control of $\mathrm{A} / \mathrm{C}$ system into the field of vehicle energy management, and tests two common optimization algorithms for a specific driving scenario. A conclusion is given in Section V.

\section{Modeling A/C System with Storage Evaporator}

As shown in Figure 1, the A/C system of a passenger car is generally based on a simple vapor compression cycle realized through a fixed-displacement rotary piston compressor, a condenser heat exchanger with a fan, a receiver/drier, an evaporator with a blower, and a thermal expansion valve. The compressor is clutched on/off to remove heat from the air flowing through the evaporator and 
reject heat into the air flowing through the condenser, determined by the blower and fan rotation speed, respectively.

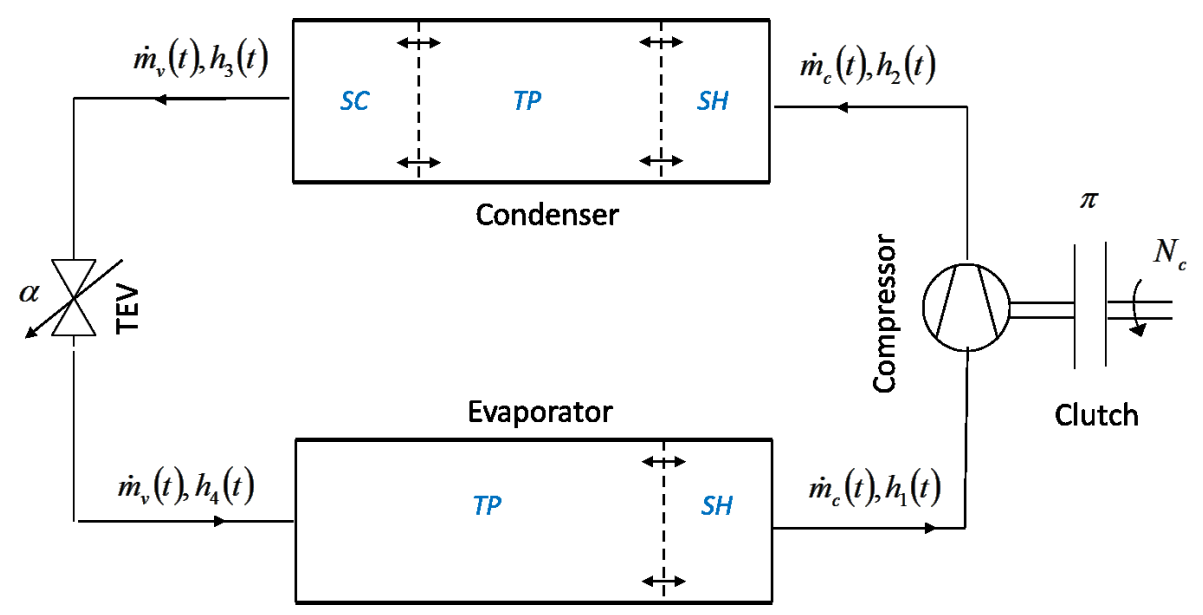

Figure 1: Conventional A/C Loop with Refrigerant Phase Change

In an innovative $\mathrm{A} / \mathrm{C}$ loop, the conventional evaporator is replaced with a storage evaporator with PCM added [9]. Physically, PCM in the outer tube is assembled around an inner tube which the refrigerant flows through. When the compressor is turned on, the refrigerant evaporates to solidify PCM that further cools down air flowing fins; when the compressor stops, the PCM starts to melt to prevent the air temperature rising up quickly until it is completely melted.

In addition to the refrigerant phase change between vapor and liquid, another phase change happens in the storage evaporator is the PCM melting or freezing between liquid and solid, both of which need to be captured in order to fully characterize the dynamics of the storage evaporator. In order to develop a supervisory controller, it is necessary to build suitable control-oriented A/C models balancing model accuracy and simulation time. Therefore, an energybased model for the A/C system with storage evaporator is developed herein with major assumptions introduced sequentially to minimize the model complexity, since the computation burdens of optimization algorithms later developed strongly rely on the total number of states within the A/C model. 


\subsection{Lumped Parameter Modeling Approache}

The methodology of building a high-fidelity A/C model is generally classified into two categories, namely finite-volume method and moving-boundary method. However, the total number of system states in both cases are much more than the level that can be tolerated by those optimization algorithms commonly adopted in vehicle energy management. The main drawback of both methods is that the physical properties are treated as distributed parameters, meaning that a group of thermodynamic variables are required to characterize the thermo-fluid dynamics inside individual control volume. Therefore, a lumped-parameter modeling approach is adopted to facilitate future optimization algorithms development.

Assumption I: the temperature spatial distribution of refrigerant, wall and PCM is uniform along the tube length direction.

Hence, the condenser is modeled as a lumped thermal mass with two control volumes representing, respectively, the volume occupied by the refrigerant circulating in the tubes and the metal mass (walls). Similarly, the evaporator consists of four control volumes, two of which are closely related to PCM dynamics. Considering the schematic of a storage evaporator with refrigerant flowing in the inner tube and PCM stored in the outer tube, as shown in Figure 2 , the spatially average PCM temperature $T_{p c m}$ and exterior wall temperature $T_{\text {ewo }}$ are determined by the heat transfer rates between refrigerant, PCM and air.

Following the assumption, an energy balance can be formulated for the PCM control volume:

$$
\frac{d}{d t}(V \rho u)=\dot{Q}_{e w i, p c m}+\dot{Q}_{p c m, e w o}
$$

where $\dot{Q}_{\text {ewi,pcm }}$ is heat transfer rate between the interior tube wall and the

PCM, and $\dot{Q}_{p c m, e w o}$ is the heat transfer rate between the PCM and the exterior tube wall.

The energy balance equation for the exterior tube wall is,

$$
M_{e w o} c_{p, e w o} \frac{d T_{e w o}}{d t}=-\dot{Q}_{p c m, e w o}-\dot{Q}_{e w o, a}
$$




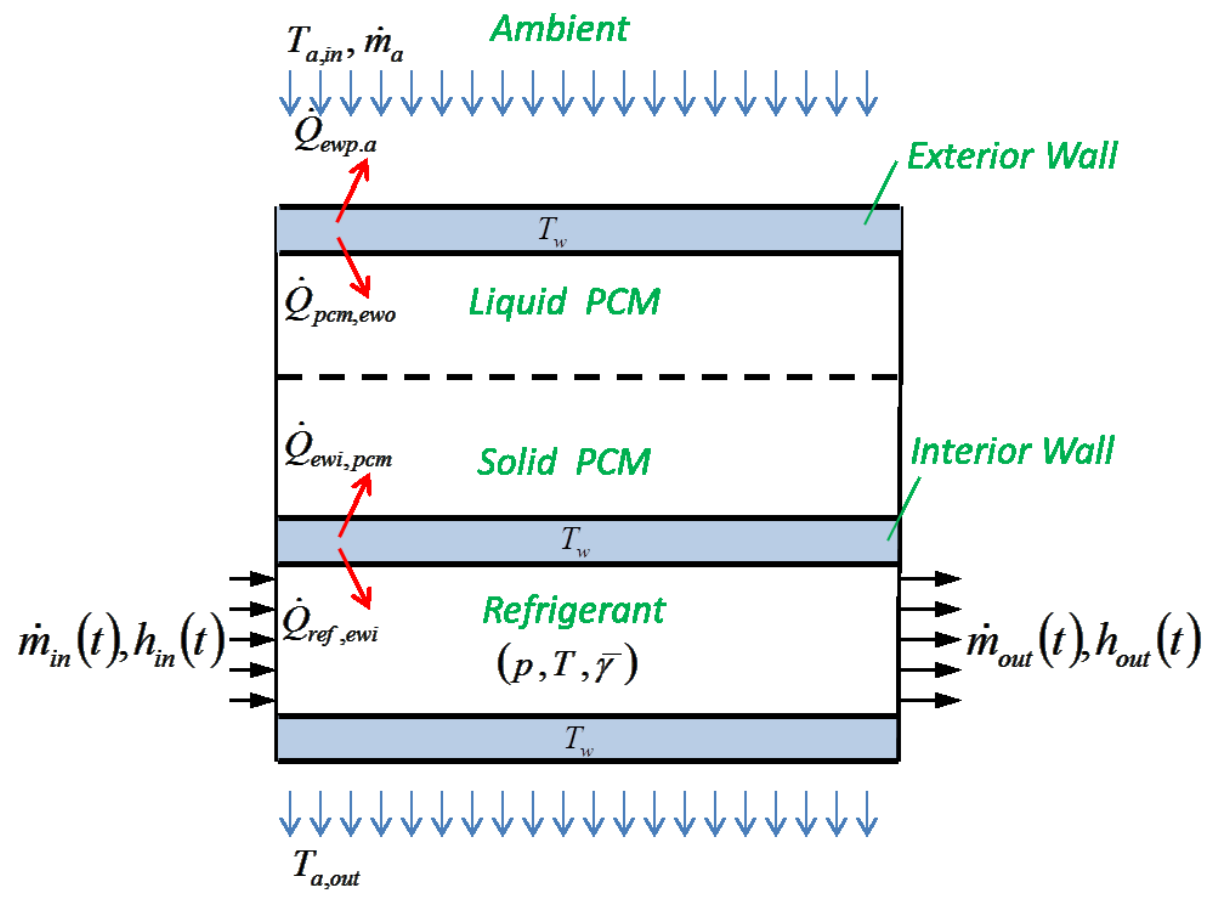

Figure 2: Schematic of Heat Exchanger with PCM included (PCM in Lower Part Is Not Drawn).

where $M_{\text {ewo }}$ is the exterior wall mass, $c_{p, \text { ewo }}$ is the exterior wall specific heat, and $\dot{Q}_{e w o, a}$ is the heat transfer rate between the exterior wall and air.

The heat transfer rate from the lumped PCM and exterior wall to the air, $\dot{Q}_{p c m, a}$, is defined similar to $\dot{Q}_{c}$, and the heat transfer rate from the refrigerant to the lumped PCM and exterior wall, $\dot{Q}_{e, p c m}$, is determined mainly by their temperature difference.

$$
\begin{aligned}
& \dot{Q}_{p c m, a}=\dot{m}_{a, e} c_{p, a}\left(T_{a, o u t, e}-T_{a, i n, e}\right) \\
& \dot{Q}_{e, p c m}=K_{p c m} A\left(T_{e}-T_{p c m}\right)
\end{aligned}
$$

in which the heat transfer coefficient $K_{p c m}$ is a piecewise function defined for each mode [15]. 


\subsection{Refrigerant Dynamics}

The dynamics on the refrigerant side involves both mass migration and heat transfer. Since the energy-based A/C model is tailored for vehicle energy management, the faster mass migration dynamics is simplified according to

Assumption II: the compliance effects relative to the variations in mass flow rate in or out of the heat exchangers are negligible [20, 21].

This assumption preserves the low-frequency dynamics, driven mainly by the heat transfer process. Hence the refrigerant mass flow rate predicted by the compressor model is uniform throughout the system. When the clutch is engaged, the mass flow rate within the $\mathrm{A} / \mathrm{C}$ loop is equal to the one calculated by the compressor model; when the clutch is disengaged, the mass flow rate within the $\mathrm{A} / \mathrm{C}$ loop is null.

The compressor is treated as an algebraic element that outputs the refrigerant flow rate and enthalpy at the exit, as function of the thermodynamic conditions at the inlet, the condenser pressure and the rotational speed:

$$
\dot{m}_{c}=V_{d} \rho_{1} \omega_{c} \eta_{v}, \quad h_{2}=h_{1}+\frac{h_{2 s}-h_{1}}{\eta_{s}}
$$

where $\omega_{c}$ is the compressor rotation speed, $V_{d}$ the compressor displacement and $h_{2 s}$ the isentropic enthalpy.

As for the heat transfer process, the pressures inside the evaporator and condenser are of interest for the high-level controller managing vehicle energy, since the mechanical power entering the A/C loop through the compressor is stored in the pressurized refrigerant. In contrast, the variation of refrigerant temperature in different phase regions is out of scope, because it is only relevant to the low-level controller that regulates superheat temperature for safety consideration. Therefore, the refrigerant dynamics can be further simplified.

Assumption III: The refrigerant within each heat exchanger is assumed present only in two-phase.

Although this represents a drastic simplification of the physical behavior, the contribution of superheat or subcooled regions to the heat transfer are in practice limited [22]. Their influences might be compensated by the calibration 
parameters in the energy-based A/C model, namely the tuning factors multiplying the heat transfer coefficients.

After mathematical derivations detailed in [2], the final energy balance equations for the refrigerant inside both evaporator and condenser are:

$$
\begin{aligned}
& V_{e}\left[\left(1-\overline{\gamma_{1}}\right) \frac{\partial\left(\rho_{l} h_{l}\right)_{e}}{\partial p_{1}}+\overline{\gamma_{1}} \frac{\partial\left(\rho_{g} h_{g}\right)_{e}}{\partial p_{1}}+\left(\rho_{g} h_{g}-\rho_{l} h_{l}\right)_{e} \frac{\partial \overline{\gamma_{1}}}{\partial p_{1}}-1+\right. \\
& \left.+\frac{M_{w e} c_{e}}{V_{e}}\left(\frac{\partial T_{1}}{\partial p_{1}}\right)\right] \frac{d p_{1}}{d t}=\dot{Q}_{e}+\pi \dot{m}\left(h_{4}-h_{1}\right) \\
& V_{c}\left[\left(1-\overline{\gamma_{2}}\right) \frac{\partial\left(\rho_{l} h_{l}\right)_{c}}{\partial p_{2}}+\overline{\gamma_{2}} \frac{\partial\left(\rho_{g} h_{g}\right)_{c}}{\partial p_{2}}+\left(\rho_{g} h_{g}-\rho_{l} h_{l}\right)_{c} \frac{\partial \overline{\gamma_{2}}}{\partial p_{2}}-1+\right. \\
& \left.+\frac{M_{w c} c_{c}}{V_{c}}\left(\frac{\partial T_{2}}{\partial p_{2}}\right)\right] \frac{d p_{2}}{d t}=-\dot{Q}_{c}+\pi \dot{m}\left(h_{2}-h_{3}\right)
\end{aligned}
$$

where the subscripts $e$ and $c$ indicate the evaporator and condenser heat exchanger, respectively.

Most of the parameters of the energy-based model can be easily related to design and geometric data of the heat exchangers, as well as to the properties of the fluids. In the single phase regions, the heat transfer correlation includes two parts [23]. For evaporation, the correlation proposed in [24] combines the nucleating boiling and convective boiling effects, $\alpha_{t p}=S \alpha_{n b}+F \alpha_{s p}$. For condensation, the correlation given by [25] combines the influences of the forced convection $N u_{F}$ and gravity controlled convection $N u_{B}$ using an asymptotic expression $N u=\left(N u_{F}^{2}+N u_{B}^{2}\right)^{1 / 2}$. In addition, since the heat transfer coefficients on the refrigerant side have been neglected due to their high value, only the air side is considered in the model. The correlations to calculate the heat transfer coefficients adopted in this work are specific to cross-flow compact heat exchangers with louvers and fins, and have been proposed by [26] and [27]. This simplifies the calibration process to identifying two multipliers, $k_{\alpha_{e, a}}$ and $k_{\alpha_{c, a}}$, which correct the heat transfer correlations for the air side of the two heat exchangers. Its ability to predict the energy consumption of the $\mathrm{A} / \mathrm{C}$ system during transients was conducted against experimental data collected on the SC03 air conditioning test cycle [2]. 


\subsection{PCM Mode Switching}

The dynamics on PCM side involves only heat transfer. One main assumption proposed in [15] is adopted to facilitate the derivation of mathematical model for the PCM.

Assumption IV: the phase change process is slow enough to consider that heat transfer in the growing phase is in the steady state, and to assume a linear temperature profile in this phase.

In other words, the sensible heat in the growing phase is negligible compared to the latent heat involved, which is justified by the small Stefan number defined as Ste $=c_{p}\left(T_{e w}-T_{p c m}\right) / L_{f}=2.59 \times 3 / 280=0.0267$.

The PCM in the storage evaporator undergoes three modes shown in Figure 3 [15], namely freezing/melting mode, frozen mode and melted mode. Depending on the modes, the left hand side of Equation (1) can be expressed in different ways.

At the freezing and melting modes, the PCM temperature $T_{p c m}$ is fixed, and is equal to the phase change temperature $T_{\text {freezing }}$ or $T_{\text {melting }}$. The latent heat caused by phase change of the PCM between solid and liquid is used to store net heat transfer, resulting the solid PCM depth $s$ varying. Hence, its energy balance equation using the solid PCM depth $s$ as a state variable is written as.

$$
\left\{\begin{array}{l}
-\rho_{p c m} L_{f} A_{c} \frac{d s}{d t}=\dot{Q}_{e, p c m}+\dot{Q}_{p c m, e w o} \\
T_{p c m}=T_{\text {freezing }}=T_{\text {melting }}
\end{array}\right.
$$

where $\rho_{p c m}$ is almost constant, $L_{f}$ is the latent heat, and $A_{c}$ is the cross sectional area of the phase change material. The solid PCM depth $s$ decreases if it absorbs heat and melts, and increases if it rejects heat and freezes. When the solid PCM depth $s$ comes to zero, it switches into the completely melted mode; when the solid PCM depth $s$ approaches the total PCM depth $e_{p c m}$, it switches into the completely frozen mode.

At the completely melted and completely frozen modes, the solid PCM depth $s$ becomes 0 or $e_{p c m}$, respectively, and the PCM is in pure liquid or solid form. 


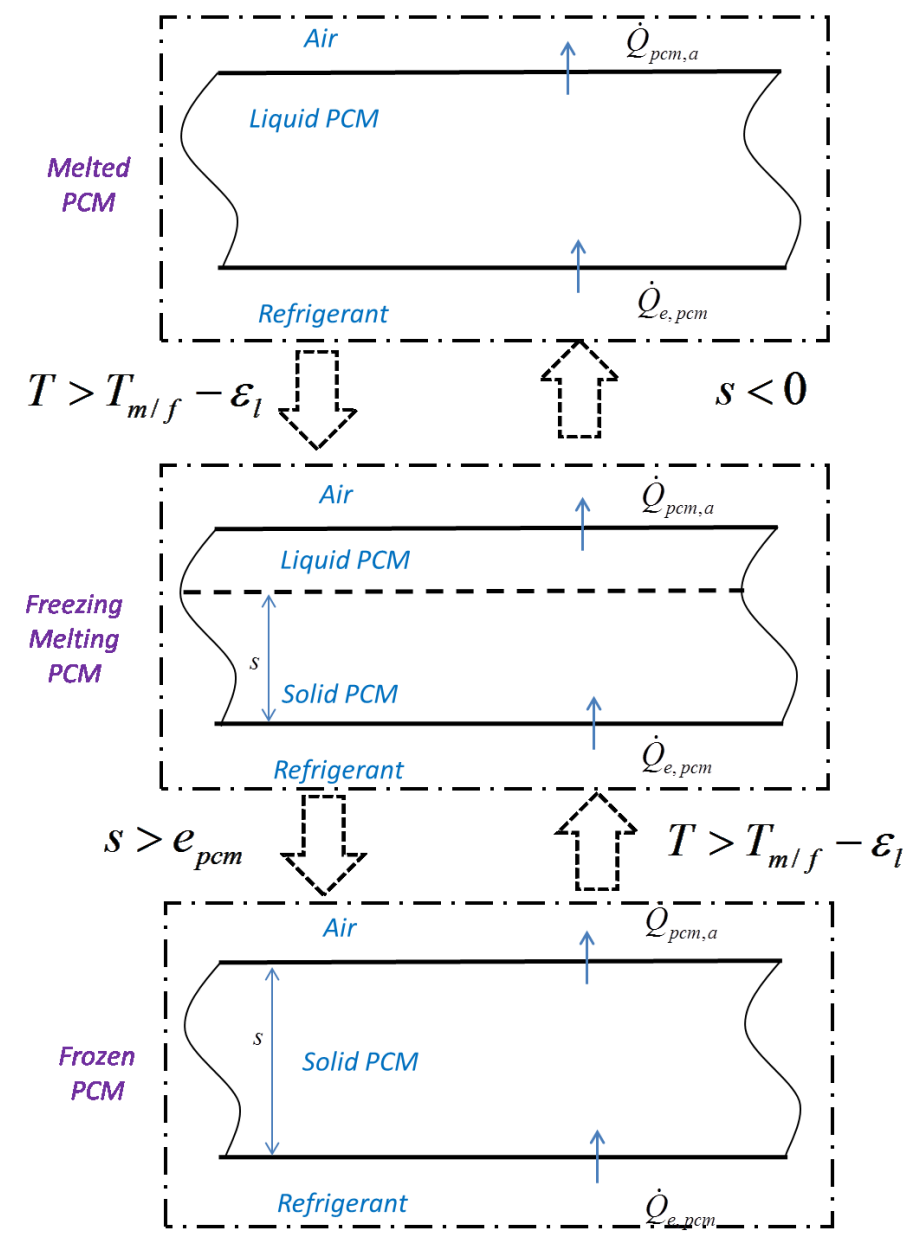

Figure 3: Modes and Mode Switching Condition 
Its temperature $T_{p c m}$ is determined by the heat transferred through the interior and exterior tube walls. The energy balance equations is,

$$
\left\{\begin{array}{l}
s=\text { constant } \\
M_{p c m} C_{p c m}^{s} \frac{d T_{p c m}}{d t}=\dot{Q}_{e, p c m}+\dot{Q}_{p c m, e w o}
\end{array}\right.
$$

where $M_{p c m}$ is PCM mass, $C_{p c m}^{s}$ is PCM specific heat. At completely melted mode, when the PCM temperature $T_{p c m}$ falls down to the the freezing temperature $T_{\text {freezing }}$, it switches from melted mode to freezing mode. At completely frozen mode, when the PCM temperature $T_{p c m}$ rises up to the melting temperature $T_{\text {melting }}$, it switches from the frozen mode to melting mode.

\subsection{Descriptor Form}

So far, the dynamics of the PCM and the exterior wall are separated. From the observation that the heat transfer dynamics is typically dominated by the fluid with the lowest convective heat transfer coefficient (the external air side), we propose the last assumption

Assumption V: The heat transfer from the PCM to the walls is assumed infinitely fast [22].

In order to transform the system into a state space form with minimum number of states, the exterior wall temperature is eliminated by lumping the wall thermal capacitance into the PCM side, because the heat transfer coefficient between the PCM and the exterior wall is much bigger than the one between the exterior wall and the air.

$$
M_{e w o} c_{p, e w o} \frac{d T_{p c m}}{d t} \approx M_{e w o} c_{p, e w o} \frac{d T_{e w o}}{d t}=-\dot{Q}_{p c m, e w o}-\dot{Q}_{e w o, a}
$$

When PCM is in melting/freezing mode, the PCM temperature $T_{p c m}$ is not changed, indicating the variation of the exterior wall temperature $T_{\text {ewo }}$ is negligible; When PCM is in melted or frozen modes, the PCM temperature $T_{p c m}$ is changing, meaning that the thermal capacitance of the exterior wall should be considered. 
Substitute Equation (8) into Equation (6), we get the differential equations describing the variation of phase change material depth when it is in freezing and melting modes, as follows:

$$
\left\{\begin{array}{l}
-\rho_{p c m} L_{f} A_{c} \frac{d s}{d t}=\dot{Q}_{e, p c m}-\dot{Q}_{p c m, a} \\
T_{p c m}=T_{\text {freezing }}=T_{\text {melting }}
\end{array}\right.
$$

Substitute Equation (8) into Equation (7), we get the differential equations describing the variation of phase change material temperature when it is melted and frozen modes, as follows:

$$
\left\{\begin{array}{l}
s=\text { constant } \\
\left(M_{p c m} C_{p c m}^{s}+M_{e w o} c_{P, e w o}\right) \frac{d T_{p c m}}{d t}=\dot{Q}_{e, p c m}-\dot{Q}_{p c m, a}
\end{array}\right.
$$

In order to merge Equation (9) and Equation (10) into one set of differential equations, logic statement is incorporated into system dynamics as suggested in Equation (11). The two forms are mathematically equivalent if a logic statement on PCM temperature is incorporated in depth dynamics and a logic statement on PCM depth is incorporated in temperature dynamics.

$$
\begin{aligned}
-\rho_{p c m} L_{f} A_{p c m} \frac{d s}{d t} & =\left[T \in\left(T_{m / f}-\epsilon_{l}, T_{m / f}+\epsilon_{u}\right)\right] \cdot\left(\dot{Q}_{e, p c m}-\dot{Q}_{p c m, a}\right) \\
\left(M_{p c m} C_{p c m}^{s}+M_{e w o} c_{p, e w o}\right) \frac{d T_{p c m}}{d t} & =\left[s \notin\left(0, e_{p c m}\right)\right] \cdot\left(\dot{Q}_{e, p c m}-\dot{Q}_{p c m, a}\right)
\end{aligned}
$$

The energy-based A/C model with a storage evaporator can be expressed in descriptor form to facilitate its implementation and its use for control design. Note that the aforementioned model combining Equation (5) and Equation (11) representative of a four-state nonlinear system and can be formulated as:

$$
\begin{aligned}
D(x) \dot{x} & =f(x, u, v) \\
y & =g(x, u, v)
\end{aligned}
$$

\section{On/Off Cycle Evaluation of Storage Evaporator}

The above storage evaporator model is implemented in Matlab/StateFlow with switching conditions labeled in Figure 3. The completely frozen and 
melt modes change to each other through an intermediate mode named melting/freezing mode. The condition indicating leaving the intermediate mode is that the solid phase change material depth s reach its physical limits, and the condition indicating entering the intermediate mode is that the phase change material temperature $T_{p c m}$ approaches the fixed phase change temperature specified by the PCM property.

Two major benefits exist if the evaporator thermal inertia is increased by adding phase change material. Firstly, the increase of discharge temperature of the storage evaporator, compared to the standard evaporator, is significantly delayed, resulting in prolonged idle-stop time of the compressor. Secondly, the losses due to the refrigerant displacement during on/off cycles are reduced since the on/off cycle period become much longer than before. Simulation results supporting the possibility of both benefits are available using thermophysical properties of a eutectic aqueous solution provided in [15] and summarized in Table 1. Various phase change temperature, phase change material thickness and thermal loads can affect the simulation results. In current simulation, all relevant parameters are chosen intentionally so that a complete melting/freezing process exists when enough on/off time period are provided.

\begin{tabular}{|c|c|c|c|}
\hline Symbol & Unit & Value & Explanation \\
\hline$L_{f}$ & $k J \mathrm{~kg}^{-1}$ & 280 & latent heat \\
\hline$\lambda_{s}$ & $W \mathrm{~m}^{-1} \mathrm{~K}^{-1}$ & 1.8 & Solid PCM thermal conductivity \\
\hline$\lambda_{l}$ & $W \mathrm{~m}^{-1} \mathrm{~K}^{-1}$ & 0.6 & Liquid PCM thermal conductivity \\
\hline$\rho_{s}$ & $\mathrm{kgm}^{-3}$ & 1042 & Solid PCM density \\
\hline$\rho_{l}$ & $\mathrm{kgm}^{-3}$ & 1115 & Liquid PCM density \\
\hline$C_{p}$ & $\mathrm{Jkg}^{-1} \mathrm{~K}^{-1}$ & 2592 & Specific heat \\
\hline
\end{tabular}

Table 1: Thermopysical properties of PCM

A complete on/off cycle is shown in Figure 5 with refrigerant temperature, phase change material temperature and discharge air temperature recorded. At the beginning of the cycle, all three temperatures are set to ambient temperature 
(here $20^{\circ} \mathrm{C}$ ). When clutch is engaged, refrigerant is propelled by the compressor into condenser and the evaporator pressure falls down, causing all three temperatures reduced. Since the melting/freezing temperature is set as $5{ }^{0} C$, the phase change material temperature does not stop dropping until it reaches this physical limit. As soon as the freezing temperature is reached, phase change material starts to freeze, maintaining the three temperatures fixed for around $40 \mathrm{sec}$. After the phase change material is completely frozen, all three temperatures fall down again till an energy balance is established among refrigerant, phase change material and air. A similar analysis might be conducted duping the clutch disengagement, expect that phase change material suppress the rapid increase of evaporator pressure. From the profile of discharge temperature, it is obvious that 40 sec delay helps impeding fast variation of discharge temperature and narrowing down its amplitude.

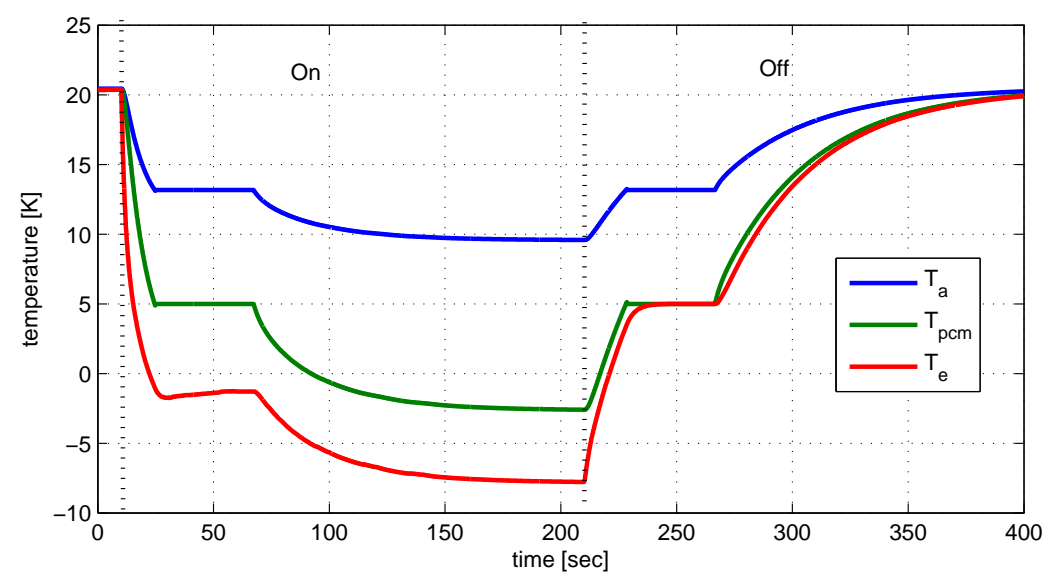

Figure 4: Temperature Profiles of Evaporator with Phase Change Material

The addition of phase change material has also an impact on the on/off periods of the A/C system. Two complete on/off cycle are presented for an advanced A/C system with storage evaporator in Figure 5 with discharge air temperature and evaporator pressure recorded. As a comparison, the on/off period of the clutch command of an ordinary A/C system without storage evaporator 
is modulated so that the average discharge air temperatures of both cases are comparable to each other. Similarly, the average evaporator pressure of both cases are maintained almost the same. From simulation results, the periodic variation of conventional evaporator pressure is more frequent the storage evaporator pressure. The on cycle of the A/C system with phase change material is five times longer and the off cycle is three time longer than the convectional A/C system. Hence, the cooling and efficiency losses caused temporary charges of the heat exchangers during on/off cycle is significantly reduced, besides compressor mechanical weariness.
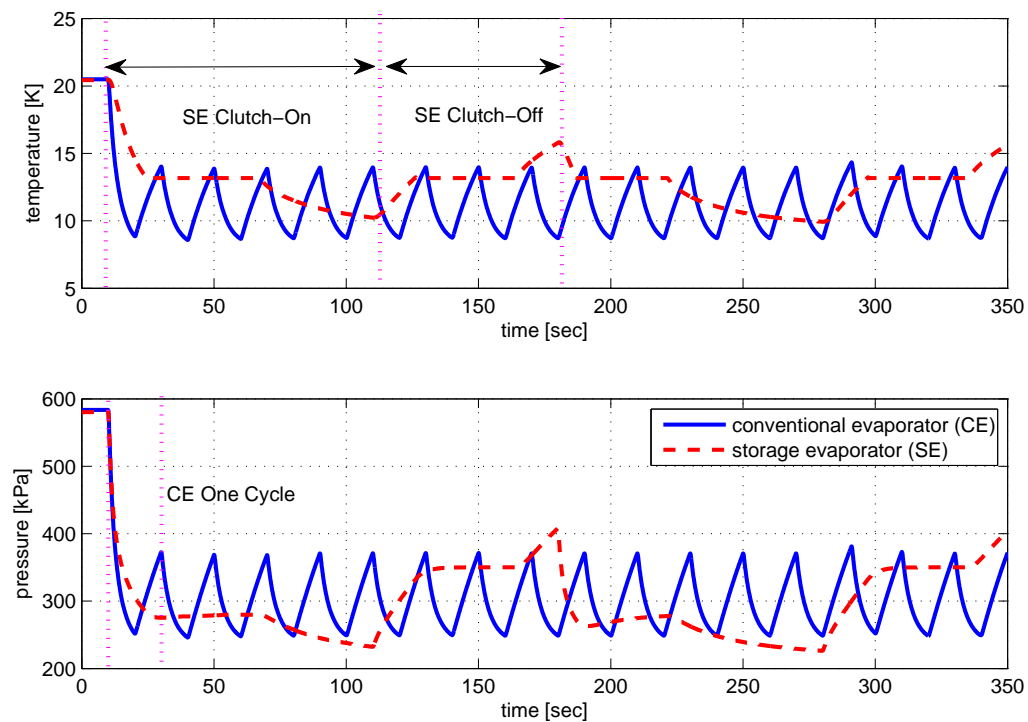

Figure 5: Evolution of Discharge Temperature and Evaporator Pressure with and without $\mathrm{PCM}$

\section{Energy Management Strategy}

The objective of a supervisory controller for the automotive A/C system is to operate the system in a way that reduces the compressor parasitic load in relation with the vehicle and engine operations, while maintaining the cabin comfort 
requirements and ensuring the reliability of the compressor clutch. Therefore the objective function for optimization problem is given by:

$$
J=\alpha J_{1}+\beta J_{2}+\gamma J_{3}
$$

where $\alpha, \beta$ and $\gamma$ are the weighting factors.

$$
\left\{\begin{array}{l}
J_{1}=\int_{0}^{T} \dot{m}_{\text {fuel }}(t) d t \\
J_{2}=\int_{0}^{T}\left(T_{1}(t)-T_{1,0}\right)^{2} d t \\
J_{3}=\int_{\Delta t}^{T}(\pi(t)-\pi(t-\tau))^{2} d t
\end{array}\right.
$$

The first term considered is the total fuel consumption over a driving profile, with $\dot{m}_{f u e l}(t)$ the instantaneous fuel consumption of the engine. The cabin comfort requirement is translated into a discharge temperature at the evaporator. Hence, a second term of the objective function represents a tracking error, Where $T_{1}$ is the actual discharge temperature and $T_{1,0}$ is a reference value for the discharge temperature. Finally, to address both drivability issues and potential durability problems, an additional cost function is considered to prevent high frequency switching in the clutch signal, where $\pi(t)$ is the current clutch position, $\pi(t-\tau)$ is the clutch position at the previous time step and $\tau$ is the discretization time. The control objective is to find the optimal trajectories of the compressor clutch command that minimize the overall objective function.

\subsection{Difficulties Faced by DP Algorithm}

DP is widely used in vehicle energy management. However, DP algorithm implemented in $[28,29]$ cannot be directly applied to the optimization problem, because it is impossible to discretize the feasible subspace over a storage evaporator model in hybrid format as explained below.

From the simulation results, it is found that the possible optimal solution, if projected from the global space $\left(p_{1}, p_{2}, s, T_{p c m}\right)$ into a subspace confined by $\left(s, T_{p c m}\right)$, always moves along the blue fold line in Figure 6. When the PCM is completely melted, $s=0$ and $T_{p c m}$ changes between a lower threshold $T_{l}$ and the phase changing temperature $T_{\text {freezing/melting }}$; when the PCM is either 
freezing or melting, $T=T_{\text {freezing }}=T_{\text {melting }}$ and $s$ changes between zero and the maximum depth $e_{p c m}$; when the PCM is completely frozen, $s=s_{p c m}$, and $T_{p c s m}$ changes between the phase changing temperature $T_{\text {freezing/melting }}$ and a upper threshold $T_{h}$. Hence, any feasible solution cannot move out of the "Z" shape domain.

In contrast, DP algorithm assumes that the feasible region is within a rectangular region outlined by $\left(s, T_{p c m}\right)=\left[0, e_{p c m}\right] \times\left[T_{l}, T_{u}\right]$. The numerical DP solver seeks to discretize the state space evenly, as depicted by the columns of red dots in Figure 6. Obviously, a PCM during freezing or melting cannot have a temperature different from phase change temperature $T_{\text {freezing/melting }}$, since it is a hard constraint imposed by its physical property. Therefore, the DP algorithm implemented in $[28,29]$ fails at the very first step when the irregular subspace $\left(s, T_{p c m}\right)$ needs gridding.

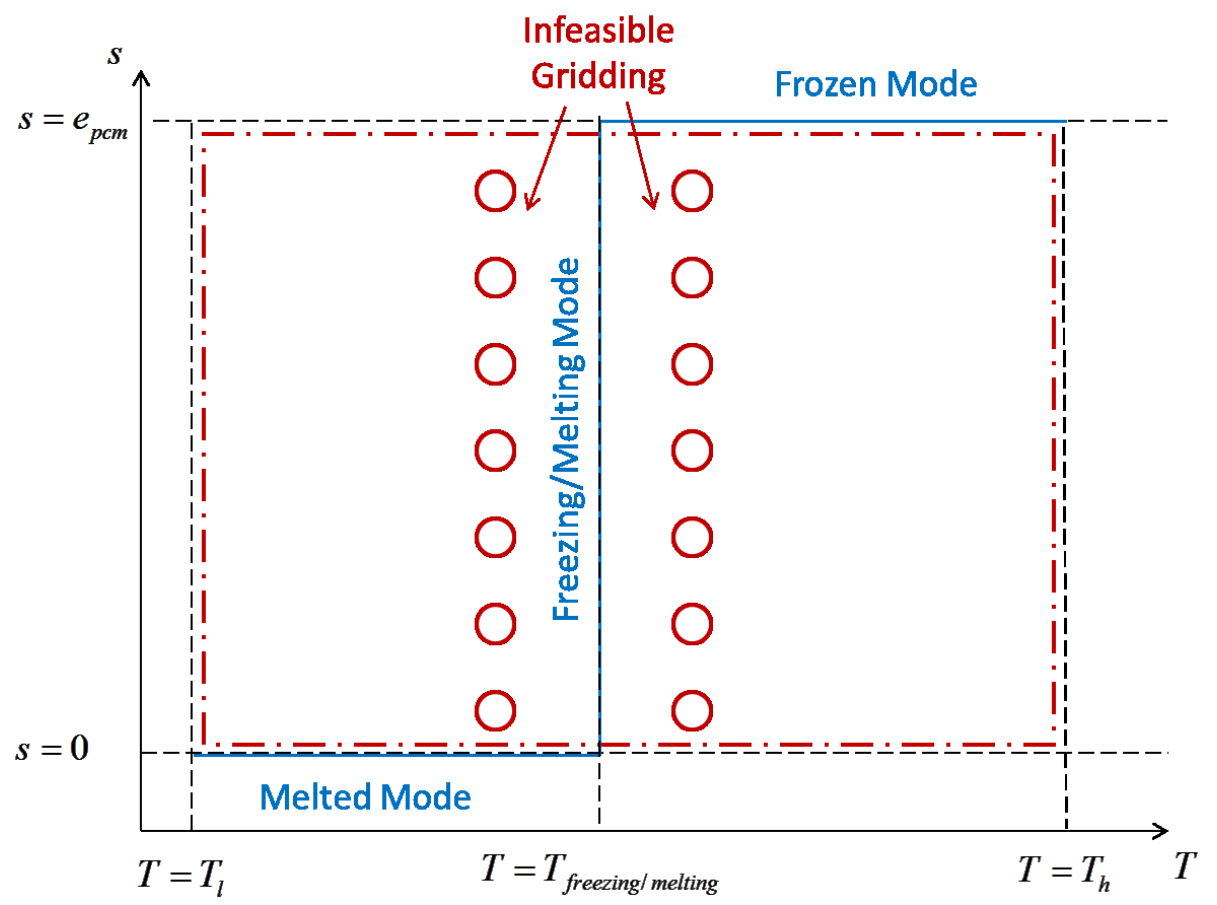

Figure 6: DP Failure Due to Discretization Difficulty 


\subsection{Hybrid Minimum Principle}

Hybrid systems are systems whose dynamics are given by a coupling of continuous variables and modes in finite sets. A switched system, as a specific type of hybrid system, consists of several subsystems and a switching law, which takes places when a certain event signal is received. An event signal may be an external signal or an internal signal generated when an internal condition for the states, inputs and/or time evolution is satisfied. An autonomous transition occurs if a switch manifold $m_{q_{j-1}, q_{j}}(x)=0$ is hit. A controlled transition occurs if there exists a discrete control input $\sigma_{q_{j-1}, q_{j}} \in \Sigma_{c}$ such that $\Gamma\left(q_{j-1}, \sigma_{q_{j-1}, q_{j}}\left(t_{j}\right)\right) \equiv \Gamma\left(q_{j-1}, \sigma_{q_{j-1}, q_{j}}\right)=q_{j}$.

The energy management of $\mathrm{A} / \mathrm{C}$ systems with storage evaporator is inherently a hybrid optimal control problem. The on/off operation sequence of the compressor clutch command is a controlled transition altering the dynamics of storing/releassing energy in the pressurized refrigerant. Besides, the mode switches among melted, melting, frozen and freezing are triggered by either hitting the physical depth of the phase change material or reaching the melting/freezing temperature. Upon these hybrid features, the problem is further complicated when the continuous actuators, such as evaporator blower and condenser fan function.

For a hybrid system, its hybrid optimal problem is usually formulated using a hybrid objective function defined as:

$$
\Sigma_{i=0}^{L} \int_{t_{i}}^{t_{i+1}} l_{q_{i}}\left(x_{q_{i}}(s), u(s)\right) d s+g\left(x_{q_{L}}\left(t_{f}\right)\right)+c(x, t)
$$

where $c(x, t)$ is a penalty on switching event. Necessary conditions for the above formulation are proposed in [30] and are summarized in detail in Table 2. Note that the Hamiltonian minimization condition over mode is no longer held. Instead, while all the continuity conditions are changed with gradients of penalty on switching events. In other words, switch might occur when the two Hamiltonians are matched, regardless of their relative values. 


\begin{tabular}{|c|c|c|}
\hline & \multicolumn{2}{|c|}{ Necessary Conditions } \\
\hline & autonomous switching & controlled switching \\
\hline Costate Dynamics & \multirow{2}{*}{\multicolumn{2}{|c|}{$\begin{array}{c}\dot{\lambda}=-\frac{\partial H_{q}(x, \lambda, u)}{\partial x} \\
\left.x^{0}, \lambda^{0}, u^{0}\right) \leq H_{q^{0}}\left(x^{0}, \lambda^{0}, v\right)\end{array}$}} \\
\hline Hamiltonian Minimization & & \\
\hline Costate Continuity & $\lambda\left(t_{j}^{-}\right)=\lambda\left(t_{j}^{+}\right)+\nabla_{x} m_{j, j+1}+\nabla_{x} c$ & $\lambda\left(t_{j}^{-}\right)=\lambda\left(t_{j}^{+}\right)+\nabla_{x} c$ \\
\hline Hamiltonian Continuity & $H\left(t_{j}^{-}\right)=H\left(t_{j}^{+}\right)+\nabla_{x} m_{j, j+1}+\nabla_{t} c$ & $H\left(t_{j}^{-}\right)=H\left(t_{j}^{+}\right)+\nabla_{t} c$ \\
\hline
\end{tabular}

Table 2: Necessary conditions when objective functions defined with penalty terms

\subsection{Preliminary Application to A/C System}

An attempt is made to apply the Hybrid Minimum Principle to the energyoptimal control of the A/C system. Some approximations are introduced at this point to simplify the mathematical complexity of the problem, with the objective of verifying the feasibility of the method to the optimal control of the A/C system. Specifically, the following assumptions are adopted to reduce the complexity while not causing significant changes to the A/C system dynamics and its hybrid optimal control formulation:

- The engine is assumed operating at constant speed and torque;

- The compressor torque is considered constant and independent of the evaporator and condenser pressure.

Based on the above, an attempt was made to apply the Hybrid Minimum Principle to the energy-optimal control of the $\mathrm{A} / \mathrm{C}$ system. If the $\mathrm{A} / \mathrm{C}$ system model is written in hybrid form, the optimization problem is now characterized by the presence of two discrete inputs, namely ON when $\pi=1$ and OFF when $\pi=0$. In order to determine the mode selection, only two Hamiltonian functions corresponding to the $\mathrm{ON} / \mathrm{OFF}$ inputs are required:

$$
\begin{aligned}
& H_{\pi=1}=L+\lambda_{1} \frac{f_{1 o n}}{d_{11}}+\lambda_{2} \frac{f_{2 o n}}{d_{22}} \\
& H_{\pi=0}=L+\lambda_{1} \frac{f_{1 o f f}}{d_{11}}+\lambda_{2} \frac{f_{2 o f f}}{d_{22}}
\end{aligned}
$$

where the Lagrangian function is the same as the one defined above, for the PMP application. 
For simplicity of notation, define:

$$
\begin{aligned}
& f_{1}= \begin{cases}f_{1 o n}=-\dot{Q}_{e}+\dot{m}\left(h_{4}-h_{1}\right), & \text { if } \pi=1 ; \\
f_{1 o f f}=-\dot{Q}_{e}, & \text { if } \pi=0 .\end{cases} \\
& f_{2}= \begin{cases}f_{2 o n}=-\dot{Q}_{c}+\dot{m}\left(h_{2}-h_{3}\right), & \text { if } \pi=1 ; \\
f_{2 o f f}=-\dot{Q}_{c}, & \text { if } \pi=0 .\end{cases}
\end{aligned}
$$

hence, the unified form of the Hamiltonian is:

$$
H=L+\lambda_{1} \frac{f_{1}}{d_{11}}+\lambda_{2} \frac{f_{2}}{d_{22}}
$$

The costate dynamics is the partial differentiation of the Hamiltonian $H$ over $p_{1}$ and $p_{2}$, respectively:

$$
\begin{aligned}
& \frac{d \lambda_{1}(t)}{d t}=-\nabla x_{1} H=-\left(\frac{\partial L}{\partial p_{1}}+\lambda_{1} \frac{\partial F_{1}}{\partial p_{1}}+\lambda_{2} \frac{\partial F_{2}}{\partial p_{1}}\right) \\
& \frac{d \lambda_{2}(t)}{d t}=-\nabla x_{2} H=-\left(\frac{\partial L}{\partial p_{2}}+\lambda_{1} \frac{\partial F_{1}}{\partial p_{2}}+\lambda_{2} \frac{\partial F_{2}}{\partial p_{2}}\right)
\end{aligned}
$$

The differentiation of $L$ is trivial because only the quadratic tracking error includes two states explicitly. However, the analytical differentiation of $F_{i}$ (defined as $d_{i i}^{-1} f$ ) is not straightforward and involves differentiation of the parameter $d_{i i}$ and of the right hand side term $f$, respectively.

$$
\frac{\partial F_{i}}{\partial p_{i}}=-\frac{1}{d_{i i}^{2}} \frac{\partial d_{i i}}{\partial p_{i}} f_{i}+\frac{1}{d_{i i}} \frac{\partial f_{i}}{\partial p_{i}}
$$

Note that the second order derivatives of the refrigerant properties with respect to the system pressures is mandatory to solve $\partial d_{i i} / \partial p_{i}$ analytically:

$$
\frac{\partial d_{i i}}{\partial p_{i}}=(1-\bar{\gamma}) \frac{d^{2} \rho_{l} h_{l}}{d p_{i}^{2}}+\bar{\gamma} \frac{d^{2} \rho_{g} h_{g}}{d p_{i}^{2}}+\left(\rho_{g} h_{g}-\rho_{l} h_{l}\right) \frac{d^{2} \bar{\gamma}_{i}}{d p_{i}^{2}}+\frac{\rho_{w} V_{w} c_{p, w}}{V_{R}} \frac{d^{2} T_{i}}{d p_{i}^{2}}
$$

On the other hand, $\partial f_{i} / \partial p_{i}$ is a piecewise function defined respectively for the cases of clutch engagement and disengagement:

$$
\frac{\partial f_{i}}{\partial p_{i}}= \begin{cases}-\frac{\partial Q_{i}}{\partial p_{i}}+\dot{m} \frac{\partial \Delta h}{\partial p_{i}}+\Delta h \frac{\partial \dot{m}}{\partial p_{i}}, & \pi=1 ; \\ -\frac{\partial Q_{i}}{\partial p_{i}}, & \pi=0 .\end{cases}
$$

where the calculation of $\partial \dot{m} / \partial p_{i}$ requires analytical differentiation of the compressor model, including the complex correlations for the isentropic efficiency and the volumetric efficiency. 
In order to avoid the significant complexity of conducting analytical differentiation of the above terms, look-up tables were generated off-line for the function $F_{i}$ over different compressor speed, condenser pressure and and evaporator pressure, from which $\partial F_{i} / \partial p_{i}$ are then calculated through numerical differentiation and smoothing techniques. An example of the surfaces obtained for these functions is given in Figure 7 for engine speed $N_{e}=1300 \mathrm{rpm}$.

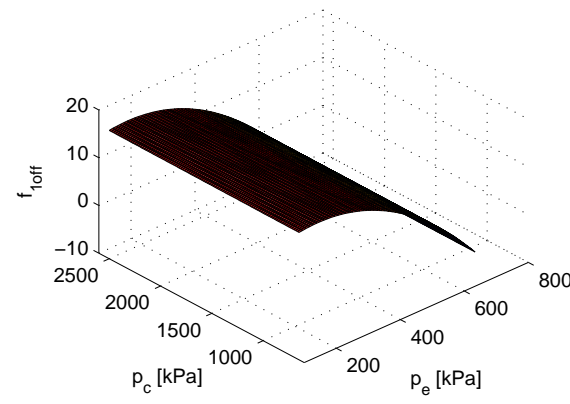

(a) Function $f_{1, o f f}$

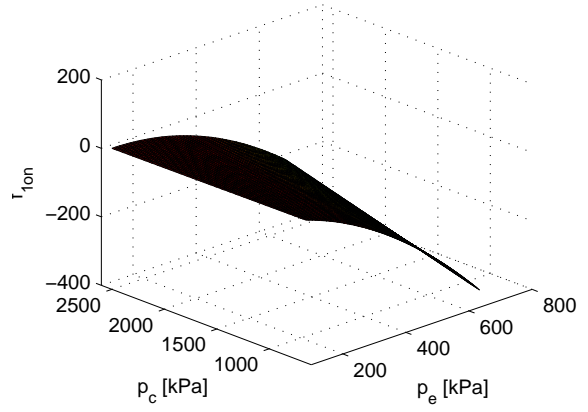

(c) Function $f_{1, \text { on }}$

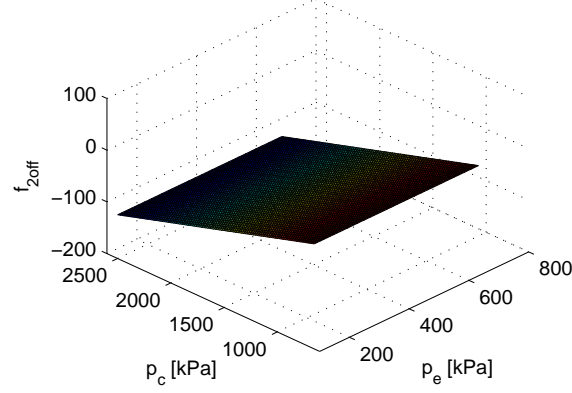

(b) Function $f_{2, o f f}$

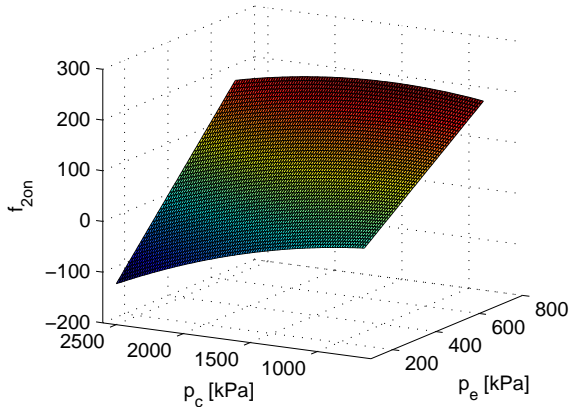

(d) Function $f_{2, o n}$

Figure 7: Example of Mapping the Nonlinear Functions of the A/C Model.

In order to apply the hybrid optimal control theory, the shooting method is required to determine the optimal value for the initial conditions of the Lagrange multipliers, $\left(\lambda_{10}, \lambda_{20}\right)$. A close observation to the optimal solution is shown in Figure 8 , limited to the first $60 \mathrm{~s}$ of simulation. The clutch command does not change the first time the two Hamiltonian functions intersect (around $5 s$ ), but 
begins switching starting from the following intersection event, until the final time is reached. This is consistent with the hybrid minimum principle stating that the Hamiltonian matching is only a necessary conditions for the clutch command to switch.
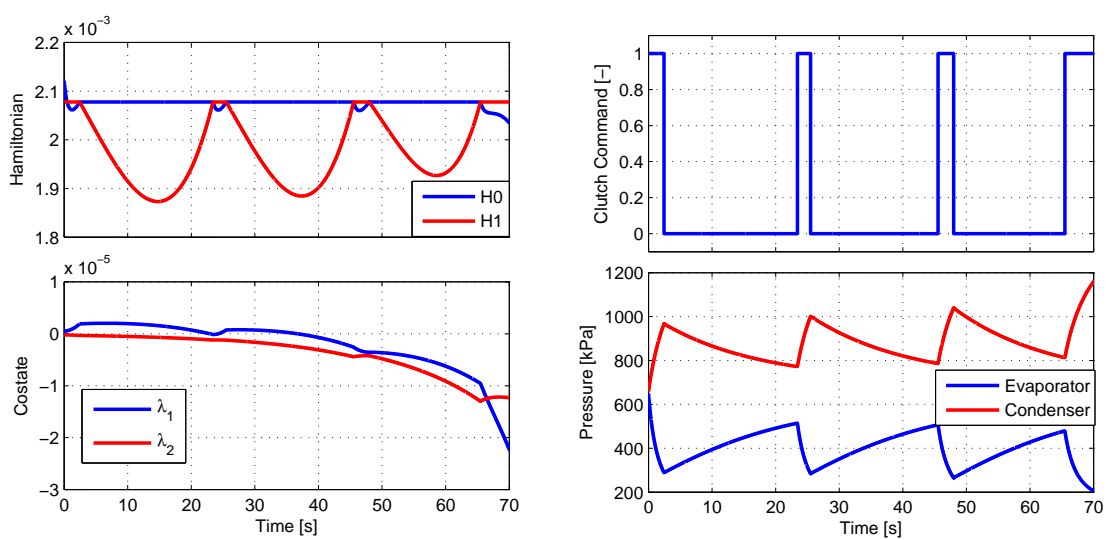

(a) Hamiltonian and Costate

(b) Clutch Command and Pressures

Figure 8: Optimal Solution of the Simplified A/C System Using HMP-IDP.

\section{Conclusion}

In this paper, an energy-based model of $\mathrm{A} / \mathrm{C}$ system with storage evaporator is developed using a lumped-parameter approach. The inherent hybrid feature of the PCM makes the resulted model multi-mode, upon which a non-switched DP algorithm cannot work. Therefore, a new approach for the optimization of an automotive A/C system has been presented, based on hybrid optimal control theory recently proposed. A preliminary study is conducted on a specific scenario when the vehicle is cruising, and simulation results show that it is very promising to generalize the method to other formulations.

\section{Acknowledgment}

The work described in the paper is in part supported by the U.S. Department of Energy, through Chrysler, LLC as the prime contractor. The authors 
gratefully acknowledge Chrysler, LLC and Dr. Timothy C. Scott for providing the data to calibrate the model and for the useful discussions.

\section{References}

[1] J. Rugh, V. Hovland, S. Anderson, Significant fuel savings and emission reductions by improving vehicle air conditioning, Mobile Air Conditioning Summit, Washington, DC.

[2] Q. Zhang, M. Canova, Lumped-parameter modeling of an automotive air conditioning system for energy optimization and management, in: ASME 2013 Dynamic Systems and Control Conference, American Society of Mechanical Engineers, 2013, pp. V001T04A003-V001T04A003.

[3] Q. Zhang, M. Canova, Energy-optimal control of an automotive air conditioning system for ancillary load reduction, Accepted by IEEE Transactions on Control System Technology.

[4] L. F. Larsen, T. Geyer, M. Morari, Hybrid model predictive control in supermarket refrigeration systems, Proceedings of 2005 IFAC world congressPraha.

[5] D. Sarabia, F. Capraro, L. F. Larsen, C. de Prada, Hybrid nmpc of supermarket display cases, Control Engineering Practice 17 (4) (2009) 428-441.

[6] C. Sonntag, A. Devanathan, S. Engell, O. Stursberg, Hybrid nonlinear modelpredictive control of a supermarket refrigeration system, in: Control Applications, 2007. CCA 2007. IEEE International Conference on, IEEE, 2007, pp. 1432-1437.

[7] B. Solberg, P. Andersen, J. M. Maciejowski, J. Stoustrup, Optimal switching control of burner setting for a compact marine boiler design, Control Engineering Practice 18 (6) (2010) 665-675.

[8] T. G. Hovgaard, S. Boyd, L. F. Larsen, J. B. Jørgensen, Nonconvex model predictive control for commercial refrigeration, International Journal of Control 86 (8) (2013) 1349-1366.

[9] R. Manski, T. Strauss, M. Kohl, M. Weinbrenner, Storage evaporators innovative ac concept for micro hybrid vehicles, SAE Technical Paper No. 2006-01-0268. 
[10] B. Zalba, J. M. Marın, L. F. Cabeza, H. Mehling, Review on thermal energy storage with phase change: materials, heat transfer analysis and applications, Applied thermal engineering 23 (3) (2003) 251-283.

[11] M. M. Farid, A. M. Khudhair, S. A. K. Razack, S. Al-Hallaj, A review on phase change energy storage: materials and applications, Energy conversion and management 45 (9) (2004) 1597-1615.

[12] F. Wang, G. Maidment, J. Missenden, R. Tozer, The novel use of phase change materials in refrigeration plant. part 1: Experimental investigation, Applied Thermal Engineering 27 (17) (2007) 2893-2901.

[13] F. Wang, G. Maidment, J. Missenden, R. Tozer, The novel use of phase change materials in refrigeration plant. part 2: Dynamic simulation model for the combined system, Applied Thermal Engineering 27 (17) (2007) 2902-2910.

[14] F. Wang, G. Maidment, J. Missenden, R. Tozer, The novel use of phase change materials in refrigeration plant. part 3: Pcm for control and energy savings, Applied Thermal Engineering 27 (17) (2007) 2911-2918.

[15] K. Azzouz, D. Leducq, D. Gobin, Performance enhancement of a household refrigerator by addition of latent heat storage, International Journal of Refrigeration 31 (5) (2008) 892-901.

[16] H. J. Sussmann, A Maximum Principle for Hybrid Optimal Control Problems, in: Decision and Control, 1999. Proceedings of the 38th IEEE Conference on, Vol. 1, IEEE, 1999, pp. 425-430.

[17] P. Riedinger, F. Kratz, C. Iung, C. Zanne, Linear Quadratic Optimization for Hybrid Systems, in: Decision and Control, 1999. Proceedings of the 38th IEEE Conference on, Vol. 3, IEEE, 1999, pp. 3059-3064.

[18] P. Riedinger, C. Zanne, F. Kratz, Time Optimal Control of Hybrid Systems, in: American Control Conference, 1999. Proceedings of the 1999, Vol. 4, IEEE, 1999, pp. $2466-2470$.

[19] M. S. Shaikh, P. Caines, On the Hybrid Optimal Control Problem: Theory and Algorithms, Automatic Control, IEEE Transactions on 52 (9) (2007) 1587-1603. 
[20] M. Browne, P. Bansal, Transient simulation of vapour-compression packaged liquid chillers, International journal of refrigeration 25 (5) (2002) 597-610.

[21] M. Kumar, I. Kar, A. Ray, State space based modeling and performance evaluation of an air-conditioning system, HVAC\&R Research 14 (5) (2008) 797-816.

[22] J. Jensen, Dynamic modeling of thermo-fluid systems, Ph.D. thesis, Technical University of DenmarkDanmarks Tekniske Universitet, Department of Energy EngineeringInstitut for Energiteknik (2003).

[23] M. Steinke, S. Kandlikar, Single-phase liquid heat transfer in microchannels, in: International Conference on Microchannels and Minichannels, 2005.

[24] W. Zhang, T. Hibiki, K. Mishima, Correlation for flow boiling heat transfer in mini-channels, International Journal of Heat and Mass Transfer 47 (26) (2004) 5749-5763.

[25] S. Koyama, K. Kuwahara, K. Nakashita, K. Yamamoto, An experimental study on condensation of refrigerant r134a in a multi-port extruded tube, International journal of refrigeration 26 (4) (2003) 425-432.

[26] Y. Chang, C. Wang, A generalized heat transfer correlation for iouver fin geometry, International Journal of heat and mass transfer 40 (3) (1997) 533-544.

[27] M. Kim, C. Bullard, Air-side thermal hydraulic performance of multi-louvered fin aluminum heat exchangers, International Journal of Refrigeration 25 (3) (2002) 390-400.

[28] O. Sundstrom, L. Guzzella, A generic dynamic programming matlab function, in: Control Applications,(CCA) \& Intelligent Control,(ISIC), 2009 IEEE, IEEE, 2009, pp. 1625-1630.

[29] O. Sundström, D. Ambühl, L. Guzzella, On implementation of dynamic programming for optimal control problems with final state constraints, Oil \& Gas Science and Technology-Revue de linstitut Français du Pétrole 65 (1) (2010) 91-102.

[30] A. Pakniyat, P. E. Caines, The Hybrid Minimum Principle in the Presence of Switching Costs, in: Decision and Control, 2013. Proceedings. 52nd IEEE Conference on, 2013. 


\author{
Nomenclature \\ TP Two Phase \\ SH Superheated \\ SC Subcooled \\ N Compressor Speed \\ $\mathrm{T}$ Temperature \\ a air \\ c Condenser \\ cmp Compressor \\ e Evaporator \\ g gas \\ h Enthalpy \\ 1 liquid \\ p Pressure \\ v Valve \\ $\dot{m}$ Mass Flow Rate \\ $\dot{Q}$ Heat Transfer Rate \\ $\alpha$ Valve Position \\ $\gamma$ Void Fraction \\ $\delta$ uncertainty \\ $\rho$ Density
}

